\title{
ON COUPLED SYSTEM OF NONLINEAR HYBRID DIFFERENTIAL EQUATION WITH ARBITRARY ORDER
}

Sajad Ali Khan, Kamal Shah*, Rahmat Ali Khan

\author{
Department of Mathematics, University of Malakand, Khyber Pakhtunkhwa, Pakistan \\ *Corresponding Author email: kamalshah408@gmail.com
}

This is an open access article distributed under the Creative Commons Attribution License, which permits unrestricted use, distribution, and reproduction in any medium, provided the original work is properly cited

\section{ARTICLE DETAILS}

\section{Article History:}

Received 5 July 2017

Accepted 8 October 2017

Available online 4 November 2017

Keywords:

Coupled system, Nonlocal boundary value problems, Fractional differential equations, linear perturbation.

\section{ABSTRACT}

This paper is devoted to the study of the existence of solution to the following toppled system:

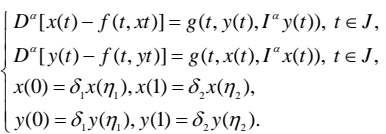

Where $D$ stands for Cupoto fractional derivative of order $\alpha$, where $1<\alpha \leq 2, J=[0,1]$, and the functions $f: J \times R \times R \rightarrow R, f(0,0)=0$ and $g: J \times R \times R \rightarrow R$ satisfy certain conditions. The proof of the existence theorem is based on a coupled fixed-point theorem of Krasnoselskii type, which extends a fixed-point theorem of Burton. Finally, our results are illustrated by a concrete example.

\section{INTRODUCTION}

Nonlinear differential equations are crucial tools in the modeling of nonlinear real phenomena corresponding to a great variety of events, in relation with several fields of the physical sciences and technology [1] For instance, they appear in the study of the air motion or the fluid dynamics, electricity, electromagnetism or the control of nonlinear process, among others [2]. The resolution of nonlinear differential equations requires, in general, the development of differential techniques in order to deduce the existence and other essential properties of the solutions. There are still many open problems related the solvability of nonlinear system, apart from the fact this is a field where advances are continuously taking place.

Perturbation techniques are useful in the nonlinear analysis for studying the dynamical systems represented by nonlinear differential and integral equations. Evidently, some differential equations representing a certain dynamical system have no analytical solution, so the perturbation of such problems can be helpful. The perturbed differential equations are categorized into various types. An important type of these such perturbations is called a hybrid differential equation (i.e quadratic perturbation of nonlinear differential equation), and the references therein [3].

Recently, the hybrid differential equations have been much more attractive, and then there have been many works on the theory of hybrid differential equations [4-7]. Additionally, hybrid fixed point theory can be used to develop the existence theory for the hybrid equations. We refer to the article [8-12]. Dhage and Jadhav discussed the following firstorder hybrid differential equation with linear perturbation of second type:

$$
\left\{\frac{d}{d t}[x(t)-f(t, x(t))]=g(t, x(t)) \text { a.e.t } \in J, x\left(t_{0}\right)=x_{0} \in R .\right.
$$

Where $J=\left[t_{0}, t_{0}+a\right]$ in $R$ for some fixed $t_{0}, a \in R$, with $a>o$, and $f, g \in C(J \times R, R)$. They proved the existence of the maximal and minimal solution for this equation [13]. Furthermore, they established some basic results concerning the strict and non-strict differential inequalities.
Indeed, the fractional differential equations have recently been intensively used in modeling of several phenomena and have been studied by many researchers in recent years, therefore they seem to deserve an independent study of their theory parallel to the theory of ordinary differential equations [14-23]. The following some problems using the differential operator in Caputo's sense were studied by some authors for existence of solutions given by

$$
\left\{\begin{array}{l}
D_{0+}^{\alpha} \phi_{p}\left(D_{0+}^{\beta} u(t)\right)=f\left(t, u(t), D_{0+}^{\beta} u(t)\right), \\
D_{0+}^{\beta} u(0)=D_{0+}^{\beta} u(1)=0,
\end{array}\right.
$$

where $D_{0+}^{\alpha}$ and $D_{0+}^{\beta}$ are Caputo's fractional derivatives, $0<\alpha, \beta \leq 1$, $1<\alpha+\beta \leq 2$. A studied the following two points boundary value problem for fractional differential equations with different boundary conditions

$$
\left\{\begin{array}{l}
D_{0+}^{\alpha} \phi_{p}\left(D_{0+}^{\beta} u(t)\right)=f\left(t, u(t), D_{0+}^{\beta} u(t)\right), \\
u(0)=0, \quad D_{0+}^{\beta} u(0)=D_{0+}^{\beta} u(1),
\end{array}\right.
$$

where $D_{0+}^{\alpha}$ and $D_{0+}^{\beta}$ are Caputo's fractional derivatives, $0<\alpha, \beta \leq 1$, $1<\alpha+\beta \leq 2$. Motivated by the work cited above, in this paper, we study the following hybrid system of fractional differential equations with linear perturbation given by

$$
\left\{\begin{array}{l}
D^{\alpha}[x(t)-f(t, x t)]=g\left(t, y(t), I^{\alpha} y(t)\right), t \in J, \\
D^{\alpha}[y(t)-f(t, y t)]=g\left(t, x(t), I^{\alpha} x(t)\right), t \in J, \\
x(0)=\delta_{1} x\left(\eta_{1}\right), x(1)=\delta_{2} x\left(\eta_{2}\right), \\
y(0)=\delta_{1} y\left(\eta_{1}\right), y(1)=\delta_{2} y\left(\eta_{2}\right) .
\end{array}\right.
$$

Where $D$ stands for Cupoto fractional derivative of order $\alpha$, where $1<\alpha \leq 2, \quad J=[0,1], \quad$ and the functions $f: J \times R \times R \rightarrow R, f(0,0)=0$ and $g: J \times R \times R \rightarrow R$ satisfy certain conditions. We study existence of at least one solution to the aforesaid problem using coupled fixed-point theorem of Burton type and its extension to receive the required results [24]. We also provide a concrete example for the demonstration of main results. 


\section{PRELIMINARIES}

Here, in this section we give some fundamental definitions and results from fractional calculus and topological degree theory. For further detailed study, we refer to $[1,3,4,28]$. Let $C(J \times R \times R, R)$ denote the class of continues functions $f: J \times R \times R \rightarrow R$ and let $\zeta(J \times R \times R, R)$ denote the class of functions $g: J \times R \times R \rightarrow R$ such that:

i. The map $t \rightarrow g(t, x, y)$ is measurable for each $x, y \in R$,

ii. The map $x \rightarrow g(t, x, y)$ is measurable for each $x \in R$,

iii. The map $y \rightarrow g(t, x, y)$ is measurable for each $y \in R$.

The class $\zeta(J \times R \times R, R)$ is called the Caratheodory class of functions on $J \times R \times R$, which are Lebesgue integrable when bounded by Lebesgue integrable function on $J$.

We need some precise definitions of the basic concepts. The following is a discussion of some of the concepts we will need.

\subsection{Definition}

The non-integer order integral of order $p \in R_{+}$of a function ${ }_{f \in L^{\prime}([a, b], R)}$ is defined as

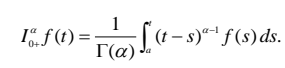

\subsection{Definition}

Let $\alpha$ be a positive number such that $m-1<\alpha<m, m \in N$ and $f^{m}(x)$ exists, a function of class $C$. Then the Caputo fractional order derivative of $f$ is defined as

$$
{ }^{c} D^{\alpha} f(x)=\frac{1}{\Gamma(m-\alpha)} \int_{0}^{t}(t-s)^{m-\alpha-1} f^{(m)}(s) d s .
$$

The following is a fixed-point theorem in Banach spaces due to Burton [1].

Lemma 2.3 The general solution to the differential equation of fractional order

$$
I^{\alpha}\left[D^{\alpha} f(t)\right]=y(t), n-1<\alpha<n,
$$

is given by

$$
I^{\alpha}\left[D^{\alpha} f(t)\right]=y(t)+c_{0}+c_{1} t+c_{2} t^{2}+\ldots+c_{m-1} t^{m-1},
$$

for arbitrary $c_{i} \in R, i=0,1,2, \ldots, m-1$.

Lemma 2.4 [1] Let $S$ be a nonempty, closed, convex, and bounded subset of a Banach space $X$ and let $A: X \rightarrow X$ and $B: S \rightarrow X$ be two operator such that

i. $A$ is a contraction with constant $\alpha<1$,

ii. $B$ is completely continues,

iii. $x=A x+B y \Rightarrow x \in S$ for all $y \in S$.

Then the operator equation $x=A x+B y$ has a solution in $S$. Now we recall the definition of a coupled fixed point for a bivariate mapping. Definition 2.5 [26] An element $(x, y) \in X \times X$ is called a coupled fixed point of a mapping $T: X \times X \rightarrow X$ if $T(x, y)=x$ and $T(y, x)=y$. Let us denoted by $\phi$ the family of all functions $\phi: R^{+} \rightarrow R^{+}$fulfilling $\phi(r)<r$ for $r>0$ and $\phi(0)=0$.

By a solution of the FHDEs system, we mean a function $(x, y) \in A C(J \times R \times R, R)$ such that:

i. the function $t \rightarrow x-f(t, x)$ is absolutely continuous for each $x \in R$, and

ii. $(x, y)$ satisfies the system of equation in (1.1)

where $A C(J, R \times R)$ is the space of absolutely continuous real-valued functions defined on $J$. Now, we prove a coupled fixed point theorem which is generalization of Lemma 2.4 of Dhage.

Theorem 2.6 Let $S$ be a nonempty, closed, convex and bounded subset of the Banach space $X$ and $\tilde{S}=S \times S$. Suppose that $A: X \rightarrow X$ and $B: S \rightarrow X$ are two operators such that

$\left(C_{1}\right)$ there exist $\phi_{A} \in \phi$ such that for all $x, y \in X$, we have

$$
\|A x-A y\| \leq \alpha \phi_{A}(\|x-y\|)
$$

for some $\sigma \geq 0$;

(C2) $B$ is completely continuous;

$\left(C_{3}\right) \quad x=A x+A y \Rightarrow x \in S$ for all $y \in S$.

Then the operator $T(x, y)=A x+A y$ has at least a coupled fixed point $\tilde{S}$ whenever $\sigma<1$.

\section{MAIN RESULT}

Throughout this section, let $X=C(J, R)$ equipped with the supremum norm $\|x\|=\sup \{|x(t)|: x(t) \in C(J)\}$. Clearly it is a Banach space with respect to point-wise operations and the supremum norm.

Now, by applying Theorem 2.6, we study the existence of solution for the FHDEs system (1.1) under the following general assumptions.

$\left(H_{0}\right) \quad$ The function $x \rightarrow x-f(t, x)$ is increasing in $R$ for all $t \in J$;

$\left(H_{1}\right) \quad$ There exists a constant $M \geq L>0$, such that

$$
|f(t, x(t))-f(t, y(t))| \leq \frac{L(\|x-y\|)}{2(M+\|x-y\|)}
$$

for all $t \in J$ and $x, y \in R$;

( $\left.H_{2}\right) \quad$ Fix $F_{0}=\max _{t \in J}|f(t, 0)|$;

$\left(H_{3}\right) \quad$ There exist a continuous function $h \in C(J, R)$ such that

$$
g(t, x(t), y(t)) \leq h(t), x, y \in R, t \in J .
$$

As a consequence of Lemma 2.3, we have the following Lemma which is useful in the existence result.

Theorem 3.1 [23] Assume that hypothesis $\left(H_{0}\right)$ holds, $y \in C(J, R), 0<p<1, \alpha>0$, and $f \in C(J \times R, R)$ with $f(0,0)=0$. Then the unique solution of the boundary value problem is given by

$$
\left\{\begin{array}{l}
x(t)=f(t, x(t))+I^{a} h(t)+\frac{\delta_{1}}{1-\delta_{1}}\left[\left(f\left(\eta_{1}, x\left(\eta_{1}\right)\right)+I^{a} h\left(\eta_{1}\right)\right.\right. \\
+\frac{\eta_{1}}{\left(1-\delta_{1}\right)\left(1-\eta_{2} \delta_{2}\right)+\left(1-\delta_{2}\right) \delta_{1}}\left[( 1 - \delta _ { 1 } ) \delta _ { 2 } \left(f\left(\eta_{2}, x\left(\eta_{2}\right)\right)+\left(1-\delta_{1}\right) \delta_{2} I^{a} h\left(\eta_{2}\right)\right.\right. \\
\left.-\left(1-\delta_{1}\right) f(1, x(1))-\left(1-\delta_{1}\right) I^{a} h\left(\eta_{1}\right)-\left(1-\delta_{2}\right) \delta_{1}\left(f\left(\eta_{1}, x\left(\eta_{1}\right)\right)-\left(1-\delta_{2}\right) \delta_{1} I^{a} h\left(\eta_{1}\right)\right]\right] \\
+\frac{\left(1-\delta_{1}\right) \delta_{2}}{\left(1-\delta_{1}\right)\left(1-\eta_{2} \delta_{2}\right)+\left(1-\delta_{2}\right) \delta_{1} \eta_{1}}\left[( 1 - \delta _ { 1 } ) \delta _ { 2 } \left(f\left(\eta_{2}, x\left(\eta_{2}\right)\right)+\left(1-\delta_{1}\right) \delta_{2} I^{a} h\left(\eta_{2}\right)\right.\right. \\
\left.-\left(1-\delta_{1}\right) f(1, x(1))-\left(1-\delta_{1}\right) I^{a} h\left(\eta_{1}\right)-\left(1-\delta_{2}\right) \delta_{1}\left(f\left(\eta_{1}, x\left(\eta_{1}\right)\right)-\left(1-\delta_{2}\right) \delta_{1} I^{a} h\left(\eta_{1}\right)\right]\right] \\
y(t)=f(t, y(t))+I^{a} h(t)+\frac{\delta_{1}}{1-\delta_{1}}\left(f\left(\eta_{1}, x\left(\eta_{1}\right)\right)\right. \\
+I^{a} h\left(\eta_{1}\right)+\frac{\eta_{1}}{\left(1-\delta_{1}\right)\left(1-\eta_{2} \delta_{2}\right)+\left(1-\delta_{2}\right) \delta_{1}} \\
{\left[( 1 - \delta _ { 1 } ) \delta _ { 2 } \left(f\left(\eta_{2}, x\left(\eta_{2}\right)\right)+\left(1-\delta_{1}\right) \delta_{2} I^{a} h\left(\eta_{2}\right)\right.\right.} \\
\left.-\left(1-\delta_{1}\right) f(1, x(1))-\left(1-\delta_{1}\right) I^{a} h\left(\eta_{1}\right)-\left(1-\delta_{2}\right) \delta_{1}\left(f\left(\eta_{1}, x\left(\eta_{1}\right)\right)-\left(1-\delta_{2}\right) \delta_{1} I^{a} h\left(\eta_{1}\right)\right]\right] \\
+\frac{\left(1-\delta_{1}\right) \delta_{2}}{\left(1-\delta_{1}\right)\left(1-\eta_{2} \delta_{2}\right)+\left(1-\delta_{2}\right) \delta_{2} \eta_{1}}\left[( 1 - \delta _ { 1 } ) \delta _ { 2 } \left(f\left(\eta_{2}, x\left(\eta_{2}\right)\right)+\left(1-\delta_{1}\right) \delta_{2} I^{a} h\left(\eta_{2}\right)\right.\right. \\
-\left(1-\delta_{1}\right) f(1, y(1))-\left(1-\delta_{1}\right) I^{a} h\left(\eta_{1}\right)-\left(1-\delta_{2}\right) \delta_{1}\left(f\left(\eta_{1}, y\left(\eta_{1}\right)\right)-\left(1-\delta_{2}\right) \delta_{1} I^{a} h\left(\eta_{1}\right)\right]
\end{array}\right.
$$

Now we are going to prove the following existence theorem for the FHDEs of system (1.1).

Theorem 3.2 Assume that hypotheses $\left(H_{1}\right)-\left(H_{3}\right)$ hold. Then the FHDEs of system has a solution defined on $J$.

Proof. Set $X=C(J, R)$ and a subset $S$ of $X$ defined by $S=[x \in X:\|x\| \leq N]$, where $M\left(L+F_{0}\right)+\frac{1}{\Gamma p+1}\|h\|_{L}^{1} \leq N$. Clearly $S$ is a nonempty, convex, closed and bounded subset of the Banach space $X$. Define two operators $A: X \rightarrow X$ and $B: S \rightarrow X$ by 


$$
\begin{aligned}
A x(t)= & f(t, x(t))+\frac{\delta_{1}}{1-\delta_{1}}\left[f\left(\eta_{1}, x\left(\eta_{1}\right)\right)\right. \\
+ & \frac{\eta_{1}}{\left(1-\delta_{1}\right)\left(1-\eta_{2} \delta_{2}\right)+\left(1-\delta_{2}\right) \delta_{1}}\left[( 1 - \delta _ { 1 } ) \delta _ { 2 } \left(f\left(\eta_{2}, x\left(\eta_{2}\right)\right)\right.\right. \\
- & \left.\left.\left(1-\delta_{1}\right) f(1, x(1))-\left(1-\delta_{2}\right) \delta_{1} f\left(\eta_{1}, x\left(\eta_{1}\right)\right)\right]\right] \\
+ & \frac{\left(1-\delta_{1}\right) \delta_{2}}{\left(1-\delta_{1}\right)\left(1-\eta_{2} \delta_{2}\right)+\left(1-\delta_{2}\right) \delta_{1} \eta_{1}}\left[( 1 - \delta _ { 1 } ) \delta _ { 2 } \left(f\left(\eta_{2}, x\left(\eta_{2}\right)\right)\right.\right. \\
- & \left.\left(1-\delta_{1}\right) f(1, x(1))-\left(1-\delta_{2}\right) \delta_{1} f\left(\eta_{1}, x\left(\eta_{1}\right)\right)\right] . \\
B x(t)= & I^{\alpha} h(t)+\frac{\delta_{1}}{1-\delta_{1}}\left[I^{\alpha} h\left(\eta_{1}\right)\right) \\
& +\frac{\left(1-\delta_{1}\right) \delta_{2}}{\left(1-\delta_{1}\right)\left(1-\eta_{2} \delta_{2}\right)+\left(1-\delta_{2}\right) \delta_{1} \eta_{1}} \\
& {\left.\left[\left(1-\delta_{1}\right) \delta_{2} I^{\alpha} h\left(\eta_{2}\right)-\left(1-\delta_{1}\right) I^{a} h\left(\eta_{1}\right)-\left(1-\delta_{2}\right) \delta_{1} I^{a} h\left(\eta_{1}\right)\right]\right] } \\
& +\frac{\delta_{1} \eta_{1}}{\left(1-\delta_{1}\right)\left[\left(1-\delta_{1}\right)\left(1-\eta_{2} \delta_{2}\right)+\left(1-\delta_{2}\right) \delta_{1}\right]} \\
& {\left[\left(1-\delta_{1}\right) \delta_{2} I^{a} h\left(\eta_{2}\right)-\left(1-\delta_{1}\right) I^{a} h\left(\eta_{1}\right)-\left(1-\delta_{2}\right) \delta_{1} I^{a} h\left(\eta_{1}\right)\right] . }
\end{aligned}
$$

So, the equation and is transformed into the system of operator equations as

$$
\begin{aligned}
& x(t)=A x(t)+B y(t), \\
& y(t)=A y(t)+B x(t) .
\end{aligned}
$$

So, we shall show that the operators $A$ and $B$ satisfy all the conditions of Theorem 2.6. Let $x, y \in X$ by hypothesis $\left(H_{1}\right)$ we have

$$
\begin{aligned}
& |A x(t)-A y(t)|=f(t, x(t))+\frac{\delta_{1}}{1-\delta_{1}}\left[\left(f\left(\eta_{1}, x\left(\eta_{1}\right)\right)+\right.\right. \\
& +\frac{\eta_{1}}{\left(1-\delta_{1}\right)\left(1-\eta_{2} \delta_{2}\right)+\left(1-\delta_{2}\right) \delta_{1}}\left[( 1 - \delta _ { 1 } ) \delta _ { 2 } \left(f\left(\eta_{2}, x\left(\eta_{2}\right)\right)\right.\right. \\
& \left.-\left(1-\delta_{1}\right) f(1, x(1))-\left(1-\delta_{2}\right) \delta_{1}\left(f\left(\eta_{1}, x\left(\eta_{1}\right)\right)\right]\right] \\
& +\frac{\left(1-\delta_{1}\right) \delta_{2}}{\left(1-\delta_{1}\right)\left(1-\eta_{2} \delta_{2}\right)+\left(1-\delta_{2}\right) \delta_{1} \eta_{1}}\left[( 1 - \delta _ { 1 } ) \delta _ { 2 } \left(f\left(\eta_{2}, x\left(\eta_{2}\right)\right)\right.\right. \\
& \left.-\left(1-\delta_{1}\right) f(1, x(1))-\left(1-\delta_{2}\right) \delta_{1}\left(f\left(\eta_{1}, x\left(\eta_{1}\right)\right)\right]\right]-f(t, y(t)) \\
& -\frac{\delta_{1}}{1-\delta_{1}}\left(f\left(\eta_{1}, x\left(\eta_{1}\right)\right)+\frac{\eta_{1}}{\left(1-\delta_{1}\right)\left(1-\eta_{2} \delta_{2}\right)+\left(1-\delta_{2}\right) \delta_{1}}\right. \\
& {\left[( 1 - \delta _ { 1 } ) \delta _ { 2 } \left(f\left(\eta_{2}, y\left(\eta_{2}\right)\right)+\left(1-\delta_{1}\right) \delta_{2} I^{a} h\left(\eta_{2}\right)\right.\right.} \\
& \left.-\left(1-\delta_{1}\right) f(1, y(1))-\left(1-\delta_{2}\right) \delta_{1}\left(f\left(\eta_{1}, y\left(\eta_{1}\right)\right)\right]\right] \\
& -\frac{\left(1-\delta_{1}\right) \delta_{2}}{\left(1-\delta_{1}\right)\left(1-\eta_{2} \delta_{2}\right)+\left(1-\delta_{2}\right) \delta_{1} \eta_{1}}\left[( 1 - \delta _ { 1 } ) \delta _ { 2 } \left(f\left(\eta_{2}, y\left(\eta_{2}\right)\right)\right.\right. \\
& -\left(1-\delta_{1}\right) f(1, y(1))-\left(1-\delta_{2}\right) \delta_{1}\left(f\left(\eta_{1}, y\left(\eta_{1}\right)\right)\right] \text {, } \\
& \leq|f(t, x(t))-f(t, y(t))|+\left|\frac{\delta_{1}}{1-\delta_{1}}\right| \mid f\left(\eta_{1}, x\left(\eta_{1}\right)-f\left(\eta_{1}, y\left(\eta_{1}\right) \mid\right.\right. \\
& +\left|\frac{\delta_{1} \eta_{1} \delta_{2}}{\left(1-\delta_{1}\right)\left(1-\eta_{2} \delta_{2}\right)+\left(1-\delta_{2}\right) \delta_{1}}\right| \mid f\left(\eta_{2}, x\left(\eta_{2}\right)-f\left(\eta_{2}, y\left(\eta_{2}\right) \mid\right.\right. \\
& +\left|\frac{\delta_{1} \eta_{1}}{\left(1-\delta_{1}\right)\left(1-\eta_{2} \delta_{2}\right)+\left(1-\delta_{2}\right) \delta_{1}}\right| \mid f(1, x(1)-f(1, y(1) \mid \\
& +\left|\frac{\delta_{1}^{2} \eta_{1}\left(1-\delta_{2}\right)}{\left(1-\delta_{1}\right)\left[\left(1-\delta_{1}\right)\left(1-\eta_{2} \delta_{2}\right)+\left(1-\delta_{2}\right) \delta_{1}\right.}\right| \mid f\left(\eta_{1}, x\left(\eta_{1}\right)-f\left(\eta_{1}, y\left(\eta_{1}\right) \mid\right.\right. \\
& +\left|\frac{\delta_{2}^{2}\left(1-\delta_{2}\right)^{2}}{\left(1-\delta_{1}\right)\left(1-\eta_{2} \delta_{2}\right)+\left(1-\delta_{2}\right) \delta_{1}}\right| \mid f\left(\eta_{2}, x\left(\eta_{2}\right)-f\left(\eta_{2}, y\left(\eta_{2}\right) \mid\right.\right. \\
& +\left|\frac{\delta_{2}\left(1-\delta_{2}\right)^{2}}{\left(1-\delta_{1}\right)\left(1-\eta_{2} \delta_{2}\right)+\left(1-\delta_{2}\right) \delta_{1}}\right| \mid f(1, x(1)-f(1, y(1) \mid \\
& +\left|\frac{\delta_{1} \delta_{2}\left(1-\delta_{1}\right)\left(1-\delta_{2}\right)}{\left(1-\delta_{1}\right)\left(1-\eta_{2} \delta_{2}\right)+\left(1-\delta_{2}\right) \delta_{1}}\right| \mid f\left(\eta_{1}, x\left(\eta_{1}\right)-f\left(\eta_{1}, y\left(\eta_{1}\right) \mid,\right.\right. \\
& \leq \frac{L(|x(t)-y(t)|)}{2(M+|x(t)-y(t)|)}+\frac{\delta_{1}}{\left(1-\delta_{1}\right)}\left[\frac{L\left(\left|x\left(\eta_{1}\right)-y\left(\eta_{1}\right)\right|\right)}{2\left(M+\left|x\left(\eta_{1}\right)-y\left(\eta_{1}\right)\right|\right)}\right] \\
& +\left(\frac{\delta_{1} \eta_{1} \delta_{2}}{\left(1-\delta_{1}\right)\left(1-\eta_{2} \delta_{2}\right)+\left(1-\delta_{2}\right) \delta_{1}}\right)\left(\frac{L\left(\left|x\left(\eta_{2}\right)-y\left(\eta_{2}\right)\right|\right)}{2\left(M+\left|x\left(\eta_{2}\right)-y\left(\eta_{2}\right)\right|\right)}\right) \\
& \begin{array}{l}
+\left(\frac{\delta_{1} \eta_{1}}{\left[1-\delta_{1}\left(1-\eta_{2} \delta_{2}\right)+\left(1-\delta_{2}\right) \delta_{1}\right]}\right)\left(\frac{\left.\left(1-\delta_{1}\right)\left(1-\eta_{2} \delta_{2}\right)+\left(1-\delta_{2}\right) \delta_{1}\right) L(|x(1)-y(1)|)}{2(M+|x(1)-y(1)|)}\right) \\
+\frac{\delta_{1}^{2} \eta_{1}\left(1-\delta_{2}\right)}{\left(1-\delta_{1}\right)\left[\left(1-\delta_{1}\right)\left(1-\eta_{2} \delta_{2}\right)+\left(1-\delta_{2}\right) \delta_{1}\right]}\left(\frac{\left.\left(1-\delta_{1}\right)\left(1-\eta_{2} \delta_{2}\right)+\left(1-\delta_{2}\right) \delta_{1}\right) L\left(\left|x\left(\eta_{1}\right)-y\left(\eta_{1}\right)\right|\right)}{2\left(M+\left|x\left(\eta_{1}\right)-y\left(\eta_{1}\right)\right|\right)}\right)
\end{array} \\
& +\frac{\delta_{2}^{2}\left(1-\delta_{1}\right)^{2}}{\left(1-\delta_{1}\right)\left[\left(1-\eta_{2} \delta_{2}\right)+\left(1-\delta_{2}\right) \delta_{1}\right]}\left(\frac{\left(\left(1-\delta_{1}\right)\left(1-\eta_{2} \delta_{2}\right)+\left(1-\delta_{2}\right) \delta_{1}\right) L\left(\left|x\left(\eta_{2}\right)-y\left(\eta_{2}\right)\right|\right)}{2\left(M+\left|x\left(\eta_{2}\right)-y\left(\eta_{2}\right)\right|\right)}\right) \\
& \left.+\frac{\delta_{2}\left(1-\delta_{1}\right)^{2}}{\left(1-\delta_{1}\right)\left[\left(1-\eta_{2} \delta_{2}\right)+\left(1-\delta_{2}\right) \delta_{1}\right]} \frac{L(|x(1)-y(1)|)}{2(M+|x(1)-y(1)|)}\right) \\
& \delta_{1}^{2}\left(1-\delta_{1}\right)\left(1-\delta_{2}\right) \\
& \left.+\frac{\delta_{2}^{2}\left(1-\delta_{1}\right)\left(1-\delta_{2}\right)}{\left(1-\delta_{1}\right)\left[\left(1-\eta_{2} \delta_{2}\right)+\left(1-\delta_{2}\right) \delta_{1}\right]} \frac{L\left(\left|x\left(\eta_{1}\right)-y\left(\eta_{1}\right)\right|\right)}{2\left(M+\left|x\left(\eta_{1}\right)-y\left(\eta_{1}\right)\right|\right)}\right),
\end{aligned}
$$

for all $t \in J$. Taking the supremum over $t$, we obtain

$$
\begin{aligned}
& \|A x(t)-A y(t)\| \leq \frac{L(\|x(t)-y(t)\|)}{2(M+\|x(t)-y(t)\|)} \\
& +\frac{\delta_{1}}{1-\delta_{1}}\left[\frac{L(\|x(t)-y(t)\|)}{2(M+\|x(t)-y(t)\|)}\right] \\
& +\frac{\delta_{1} \eta_{1} \delta_{2}}{\left(1-\delta_{1}\right)\left(1-\eta_{2} \delta_{2}\right)+\left(1-\delta_{2}\right) \delta_{1}} \\
& L(\|x(t)-y(t)\|) \\
& \left(\frac{L}{2(M+\|x(t)-y(t)\|)}\right) \\
& +\frac{\delta_{1} \eta_{1}}{\left(1-\delta_{1}\right)\left(1-\eta_{2} \delta_{2}\right)+\left(1-\delta_{2}\right) \delta_{1}} \\
& \left(\frac{\left(\left(1-\delta_{1}\right)\left(1-\eta_{2} \delta_{2}\right)+\left(1-\delta_{2}\right) \delta_{1}\right) L(\|x(t)-y(t)\|)}{2(M+\|x(t)-y(t)\|)}\right. \\
& +\frac{\delta_{1}^{2} \eta_{1}\left(1-\delta_{2}\right)}{\left(1-\delta_{1}\left[1-\delta_{1}\right)\left(1-\eta_{2} \delta_{2}\right)+\left(1-\delta_{2}\right) \delta_{1}\right]} \times \\
& \underline{\left(\left(1-\delta_{1}\left[1-\delta_{1}\right)\left(1-\eta_{2} \delta_{2}\right)+\left(1-\delta_{2}\right) \delta_{1}\right]\right) L(\|x(t)-y(t)\|)} \\
& 2(M+\|x(t)-y(t)\|) \\
& +\frac{\delta_{2}^{2}\left(1-\delta_{1}\right)^{2}}{\left.\left[1-\delta_{1}\right)\left(1-\eta_{2} \delta_{2}\right)+\left(1-\delta_{2}\right) \delta_{1}\right]} \times \\
& \frac{\left.\left(\left[1-\delta_{1}\right)\left(1-\eta_{2} \delta_{2}\right)+\left(1-\delta_{2}\right) \delta_{1}\right]\right) L(\|x(t)-y(t)\|)}{2(M+\|x(t)-y(t)\|)} \\
& +\frac{\delta_{2}\left(1-\delta_{1}\right)^{2}}{\left.\left[1-\delta_{1}\right)\left(1-\eta_{2} \delta_{2}\right)+\left(1-\delta_{2}\right) \delta_{1}\right]} \frac{L(\|x(t)-y(t)\|)}{2(M+\|x(t)-y(t)\|)} \\
& +\frac{\delta_{2}^{2}\left(1-\delta_{1}\right)\left(1-\delta_{2}\right)}{\left.\left[1-\delta_{1}\right)\left(1-\eta_{2} \delta_{2}\right)+\left(1-\delta_{2}\right) \delta_{1}\right]} \frac{L(\|x(t)-y(t)\|)}{2(M+\|x(t)-y(t)\|)}, \\
& =\left[1+\frac{\delta_{1}}{1-\delta_{1}}+\frac{\delta_{1} \eta_{1} \delta_{2}}{\left(1-\delta_{1}\right)\left(1-\eta_{2} \delta_{2}\right)+\left(1-\delta_{2}\right) \delta_{1}}\right. \\
& +\frac{\delta_{1} \eta_{1}\left(\left(1-\delta_{1}\right)\left(1-\eta_{2} \delta_{2}\right)+\left(1-\delta_{2}\right) \delta_{1}\right)}{\left(1-\delta_{1}\right)\left(1-\eta_{2} \delta_{2}\right)+\left(1-\delta_{2}\right) \delta_{1}} \\
& +\frac{\delta_{1}^{2} \eta_{1}\left(1-\delta_{2}\right)\left(\left(1-\delta_{1}\left[1-\delta_{1}\right)\left(1-\eta_{2} \delta_{2}\right)+\left(1-\delta_{2}\right) \delta_{1}\right]\right)}{\left(1-\delta_{1}\left[1-\delta_{1}\right)\left(1-\eta_{2} \delta_{2}\right)+\left(1-\delta_{2}\right) \delta_{1}\right]} \\
& +\frac{\left.\delta_{2}^{2}\left(1-\delta_{1}\right)^{2}\left(\left[1-\delta_{1}\right)\left(1-\eta_{2} \delta_{2}\right)+\left(1-\delta_{2}\right) \delta_{1}\right]\right)}{\left.\left[1-\delta_{1}\right)\left(1-\eta_{2} \delta_{2}\right)+\left(1-\delta_{2}\right) \delta_{1}\right]} \\
& +\frac{\delta_{2}\left(1-\delta_{1}\right)^{2}}{\left.\left[1-\delta_{1}\right)\left(1-\eta_{2} \delta_{2}\right)+\left(1-\delta_{2}\right) \delta_{1}\right]} \\
& \left.+\frac{\delta_{2}^{2}\left(1-\delta_{1}\right)\left(1-\delta_{2}\right)}{\left.\left[1-\delta_{1}\right)\left(1-\eta_{2} \delta_{2}\right)+\left(1-\delta_{2}\right) \delta_{1}\right]}\right] \frac{L(\|x(t)-y(t)\|)}{2(M+\|x(t)-y(t)\|)} \text {. }
\end{aligned}
$$

So

$$
\|A x(t)-A y(t)\| \leq \frac{M_{2} L(\|x-y\|)}{2(M+\|x-y\|)} .
$$

Where

$$
\begin{aligned}
& M_{2}=\left[1+\frac{\delta_{1}}{1-\delta_{1}}+\frac{\delta_{1} \eta_{1} \delta_{2}}{\left(1-\delta_{1}\right)\left(1-\eta_{2} \delta_{2}\right)+\left(1-\delta_{2}\right) \delta_{1}}\right. \\
& +\frac{\delta_{1} \eta_{1}\left(\left(1-\delta_{1}\right)\left(1-\eta_{2} \delta_{2}\right)+\left(1-\delta_{2}\right) \delta_{1}\right)}{\left(1-\delta_{1}\right)\left(1-\eta_{2} \delta_{2}\right)+\left(1-\delta_{2}\right) \delta_{1}} \\
& +\frac{\delta_{1}^{2} \eta_{1}\left(1-\delta_{2}\right)\left(\left(1-\delta_{1}\left[1-\delta_{1}\right)\left(1-\eta_{2} \delta_{2}\right)+\left(1-\delta_{2}\right) \delta_{1}\right]\right)}{\left(1-\delta_{1}\left[1-\delta_{1}\right)\left(1-\eta_{2} \delta_{2}\right)+\left(1-\delta_{2}\right) \delta_{1}\right]} \\
& +\frac{\left.\delta_{2}^{2}\left(1-\delta_{1}\right)^{2}\left(\left[1-\delta_{1}\right)\left(1-\eta_{2} \delta_{2}\right)+\left(1-\delta_{2}\right) \delta_{1}\right]\right)}{\left.\left[1-\delta_{1}\right)\left(1-\eta_{2} \delta_{2}\right)+\left(1-\delta_{2}\right) \delta_{1}\right]} \\
& +\frac{\delta_{2}\left(1-\delta_{1}\right)^{2}}{\left.\left[1-\delta_{1}\right)\left(1-\eta_{2} \delta_{2}\right)+\left(1-\delta_{2}\right) \delta_{1}\right]} \\
& \left.+\frac{\delta_{2}^{2}\left(1-\delta_{1}\right)\left(1-\delta_{2}\right)}{\left.\left[1-\delta_{1}\right)\left(1-\eta_{2} \delta_{2}\right)+\left(1-\delta_{2}\right) \delta_{1}\right]}\right]>0 .
\end{aligned}
$$

This show that $A$ is a non-linear contraction on $X$ with a control function $\frac{1}{2} \phi$ where $\phi$ is defined by $\phi(r)=\frac{L r}{M+r}$.

Next, we show that $B$ is compact and continuous operator on $S$. Let $x_{n}$ be a sequence in $S$ converging to a point $x \in S$. Then 
$\lim _{n \rightarrow \infty} B x_{n}(t)=\lim _{n \rightarrow \infty}\left[I^{\alpha} g\left(t, x_{n}(t), I^{\alpha} x(t)\right)\right.$

$$
\begin{aligned}
& +\frac{\delta_{1}}{1-\delta_{1}}\left(I^{\alpha} g\left(\eta_{1}, x_{n}\left(\eta_{1}\right), I^{\alpha} x_{n}\left(\eta_{1}\right)\right)\right. \\
& +\frac{\left(1-\delta_{1}\right) \delta_{2}}{\left(1-\delta_{1}\right)\left(1-\eta_{2} \delta_{2}\right)+\left(1-\delta_{2}\right) \delta_{1} \eta_{1}} \\
& {\left[\left(1-\delta_{1}\right) \delta_{2} I^{a} g\left(\eta_{2}, x_{n}\left(\eta_{2}\right), I^{\alpha} x_{n}\left(\eta_{2}\right)\right)\right.} \\
& -\left(1-\delta_{1}\right) I^{\alpha} g\left(\eta_{1}, x_{n}\left(\eta_{1}\right), I^{\alpha} x_{n}\left(\eta_{1}\right)\right) \\
& \left.-\left(1-\delta_{2}\right) \delta_{1} I^{\alpha} g\left(\eta_{1}, x_{n}\left(\eta_{1}\right), I^{\alpha} x_{n}\left(\eta_{1}\right)\right)\right] \\
& +\frac{\delta_{1} \eta_{1}}{\left(1-\delta_{1}\right)\left[\left(1-\delta_{1}\right)\left(1-\eta_{2} \delta_{2}\right)+\left(1-\delta_{2}\right) \delta_{1}\right]} \\
& {\left[\left(1-\delta_{1}\right) \delta_{2} I^{\alpha} g\left(\eta_{2}, x_{n}\left(\eta_{2}\right), I^{\alpha} x_{n}\left(\eta_{2}\right)\right)\right.} \\
& -\left(1-\delta_{1}\right) I^{\alpha} g\left(\eta_{1}, x_{n}\left(\eta_{1}\right), I^{\alpha} x_{n}\left(\eta_{1}\right)\right) \\
& \left.\left.-\left(1-\delta_{2}\right) \delta_{1} I^{\alpha} g\left(\eta_{1}, x_{n}\left(\eta_{1}\right), I^{\alpha} x_{n}\left(\eta_{1}\right)\right)\right]\right] \\
& =I^{\alpha} \lim _{n \rightarrow \infty} g\left(t, x_{n}(t), I^{\alpha} x_{n}(t)\right. \\
& +\frac{\delta_{1}}{1-\delta_{1}}\left(I^{\alpha} \lim _{n \rightarrow \infty} g\left(\eta_{1}, x_{n}\left(\eta_{1}\right), I^{\alpha} x_{n}\left(\eta_{1}\right)\right)\right. \\
& +\frac{\left(1-\delta_{1}\right) \delta_{2}}{\left(1-\delta_{1}\right)\left(1-\eta_{2} \delta_{2}\right)+\left(1-\delta_{2}\right) \delta_{1} \eta_{1}} \\
& {\left[\left(1-\delta_{1}\right) \delta_{2} I^{\alpha} \lim _{n \rightarrow \infty} g\left(\eta_{2}, x_{n}\left(\eta_{2}\right), I^{\alpha} x_{n}\left(\eta_{2}\right)\right)\right.} \\
& -\left(1-\delta_{1}\right) I^{\alpha} \lim _{n \rightarrow \infty} g\left(\eta_{1}, x_{n}\left(\eta_{1}\right), I^{\alpha} x_{n}\left(\eta_{1}\right)\right) \\
& \left.-\left(1-\delta_{2}\right) \delta_{1} I^{\alpha} \lim _{n \rightarrow \infty} g\left(\eta_{1}, x_{n}\left(\eta_{1}\right), I^{\alpha} x_{n}\left(\eta_{1}\right)\right)\right] \\
& +\frac{\delta_{1} \eta_{1}}{\left(1-\delta_{1}\right)\left[\left(1-\delta_{1}\right)\left(1-\eta_{2} \delta_{2}\right)+\left(1-\delta_{2}\right) \delta_{1}\right]} \\
& {\left[\left(1-\delta_{1}\right) \delta_{2} I^{\alpha} \lim _{n \rightarrow \infty} g\left(\eta_{2}, x_{n}\left(\eta_{2}\right), I^{\alpha} x_{n}\left(\eta_{2}\right)\right)\right.} \\
& -\left(1-\delta_{1}\right) I^{\alpha} \lim _{n \rightarrow \infty} g\left(\eta_{1}, x_{n}\left(\eta_{1}\right), I^{\alpha} x_{n}\left(\eta_{1}\right)\right) \\
& \left.\left.-\left(1-\delta_{2}\right) \delta_{1} I^{\alpha} \lim _{n \rightarrow \infty} g\left(\eta_{1}, x_{n}\left(\eta_{1}\right), I^{\alpha} x_{n}\left(\eta_{1}\right)\right)\right]\right] \\
& =I^{\alpha} h(t)+\frac{\delta_{1}}{1-\delta_{1}}\left(I^{\alpha} h\left(\eta_{1}\right)\right) \\
& +\frac{\left(1-\delta_{1}\right) \delta_{2}}{\left(1-\delta_{1}\right)\left(1-\eta_{2} \delta_{2}\right)+\left(1-\delta_{2}\right) \delta_{1} \eta_{1}} \\
& {\left[\left(1-\delta_{1}\right) \delta_{2} I^{\alpha} h\left(\eta_{2}\right)-\left(1-\delta_{1}\right) I^{\alpha} h\left(\eta_{1}\right)\right.} \\
& \left.-\left(1-\delta_{2}\right) \delta_{1} I^{\alpha} h\left(\eta_{1}\right)\right] \\
& +\frac{\delta_{1} \eta_{1}}{\left(1-\delta_{1}\right)\left[\left(1-\delta_{1}\right)\left(1-\eta_{2} \delta_{2}\right)+\left(1-\delta_{2}\right) \delta_{1}\right]} \\
& {\left[\left(1-\delta_{1}\right) \delta_{2} I^{\alpha} h\left(\eta_{2}\right)-\left(1-\delta_{1}\right) I^{\alpha} h\left(\eta_{1}\right)\right.} \\
& \left.-\left(1-\delta_{2}\right) \delta_{1} I^{\alpha} h\left(\eta_{1}\right)\right] \\
& =B_{x}(t) \text {, }
\end{aligned}
$$

So, for all $t \in J$, where the second equality holds by Lebesgue dominated convergent theorem. So $B$ is a continuous function on $S$. Let $x \in S$, by assumption (H2), for $t \in J$. We have

$$
\begin{aligned}
& |B x(t)|=\mid I^{\alpha} g\left(t, x(t), I^{\alpha} x(t)\right)+\frac{\delta_{1}}{1-\delta_{1}}\left(I^{\alpha} g\left(\eta_{1}, x\left(\eta_{1}\right), I^{\alpha} x\left(\eta_{1}\right)\right)\right. \\
& +\frac{\left(1-\delta_{1}\right) \delta_{2}}{\left(1-\delta_{1}\right)\left(1-\eta_{2} \delta_{2}\right)+\left(1-\delta_{2}\right) \delta_{1} \eta_{1}}\left[\left(1-\delta_{1}\right) \delta_{2} I^{a} g\left(\eta_{2}, x\left(\eta_{2}\right), I^{a} x\left(\eta_{2}\right)\right)\right. \\
& \left.-\left(1-\delta_{1}\right) I^{\alpha} g\left(\eta_{1}, x\left(\eta_{1}\right), I^{\alpha} x\left(\eta_{1}\right)\right)-\left(1-\delta_{2}\right) \delta_{1} I^{\alpha} g\left(\eta_{1}, x\left(\eta_{1}\right), I^{\alpha} x\left(\eta_{1}\right)\right)\right] \\
& +\frac{\delta_{1} \eta_{1}}{\left(1-\delta_{1}\right)\left[\left(1-\delta_{1}\right)\left(1-\eta_{2} \delta_{2}\right)+\left(1-\delta_{2}\right) \delta_{1}\right]}\left[\left(1-\delta_{1}\right) \delta_{2} I^{\alpha} g\left(\eta_{2}, x\left(\eta_{2}\right), I^{\alpha} x\left(\eta_{2}\right)\right)\right. \\
& \left.\left.-\left(1-\delta_{1}\right) I^{\alpha} g\left(\eta_{1}, x\left(\eta_{1}\right), I^{\alpha} x\left(\eta_{1}\right)\right)-\left(1-\delta_{2}\right) \delta_{1} I^{\alpha} g\left(\eta_{1}, x\left(\eta_{1}\right), I^{\alpha} x\left(\eta_{1}\right)\right)\right]\right] \mid \\
& \leq \mid\left[I ^ { \alpha } g ( t , x ( t ) , I ^ { \alpha } x ( t ) ) | + | \frac { \delta _ { 1 } } { 1 - \delta _ { 1 } } \left(I^{\alpha} g\left(\eta_{1}, x\left(\eta_{1}\right), I^{\alpha} x\left(\eta_{1}\right)\right) \mid\right.\right. \\
& +\mid \frac{\left(1-\delta_{1}\right) \delta_{2}}{\left(1-\delta_{1}\right)\left(1-\eta_{2} \delta_{2}\right)+\left(1-\delta_{2}\right) \delta_{1} \eta_{1}}\left[\left(1-\delta_{1}\right) \delta_{2} I^{a} g\left(\eta_{2}, x\left(\eta_{2}\right), I^{a} x\left(\eta_{2}\right)\right) \mid\right. \\
& \left.+\left|\left(1-\delta_{1}\right) I^{\alpha} g\left(\eta_{1}, x\left(\eta_{1}\right), I^{\alpha} x\left(\eta_{1}\right)\right)\right|+\mid\left(1-\delta_{2}\right) \delta_{1} I^{a} g\left(\eta_{1}, x\left(\eta_{1}\right), I^{\alpha} x\left(\eta_{1}\right)\right)\right] \mid \\
& +\mid \frac{\delta_{1} \eta_{1}}{\left(1-\delta_{1}\right)\left[\left(1-\delta_{1}\right)\left(1-\eta_{2} \delta_{2}\right)+\left(1-\delta_{2}\right) \delta_{1}\right]}\left[\left(1-\delta_{1}\right) \delta_{2} I^{\alpha} g\left(\eta_{2}, x\left(\eta_{2}\right), I^{x} x\left(\eta_{2}\right)\right) \mid\right. \\
& \left.\left.+\left|\left(1-\delta_{1}\right) I^{\alpha} g\left(\eta_{1}, x\left(\eta_{1}\right), I^{\alpha} x\left(\eta_{1}\right)\right)\right|+\mid\left(1-\delta_{2}\right) \delta_{1} I^{a} g\left(\eta_{1}, x\left(\eta_{1}\right), I^{\alpha} x\left(\eta_{1}\right)\right)\right]\right] \mid \\
& \leq \frac{1}{\Gamma(\alpha+1)}\|h\|_{L}^{1}+\mid \frac{\delta_{1}}{1-\delta_{1}}\left[\left[\frac{\eta_{1}^{p}}{\Gamma(\alpha+1)}\right]\|h\|_{L}^{\prime}\right. \\
& +\left|\frac{\left(1-\delta_{1}^{2}\right) \delta_{2}^{2}}{\left(1-\delta_{1}\right)\left(1-\eta_{2} \delta_{2}\right)+\left(1-\delta_{2}\right) \delta_{1} \eta_{1}}\right|\left[\frac{\eta_{2}^{p}}{\Gamma(\alpha+1)}\|h\|_{L}^{\prime}\right] \\
& +\left|\left(1-\delta_{1}\right)\right|\left[\frac{\eta_{1}^{p}}{\Gamma(\alpha+1)}\right]\left|h\left\|_{L}^{1}+\left|\left(1-\delta_{2}\right) \delta_{1}\right|\left[\frac{\eta_{1}^{p}}{\Gamma(\alpha+1)}\right]\right\| h \|_{L}^{\prime}\right. \\
& +\left|\frac{\delta_{1_{1}} \delta_{2}}{\left(1-\delta_{1}\right)\left(1-\eta_{2} \delta_{2}\right)+\left(1-\delta_{2}\right) \delta_{1} \eta_{1}}\right|\left[\frac{\eta_{2}^{p}}{\Gamma(\alpha+1)}\right]\|h\|_{2}^{\prime} \\
& +\left|\left(1-\delta_{1}\right)\right|\left[\frac{\eta_{1}^{p}}{\Gamma(\alpha+1)}\right]\|h\|_{L}^{1}+\left|\left(1-\delta_{2}\right) \delta_{1}\right|\left[\frac{\eta_{1}^{p}}{\Gamma(\alpha+1)}\right]\|h\|_{L}^{1} \\
& \left.=\frac{1}{\Gamma(\alpha+1)}\|h\|_{L}^{\prime}+\left(\left|\frac{\delta_{1}}{1-\delta_{1}}\right|+2\left(1-\delta_{1}\right)+2\left(1-\delta_{2}\right) \delta_{1}\right)\left[\frac{\eta_{1}^{p}}{\Gamma(\alpha+1)}\right]\|h\|_{L}^{\prime}\right] \\
& +\left(\left|\frac{\left(1-\delta_{1}\right)^{2} \delta_{2}^{2}}{\left(1-\delta_{1}\right)\left(1-\eta_{2} \delta_{2}\right)+\mid\left(1-\delta_{2}\right) \delta_{1} \eta_{1}}\right|+\left|\frac{\delta_{1} \eta_{1} \delta_{2}}{\left[\left(1-\delta_{1}\right)\left(1-\eta_{2} \delta_{2}\right)+\left(1-\delta_{2}\right) \delta_{1}\right]}\right|\right)\left[\frac{\eta_{2}^{p}}{\Gamma(\alpha+1)}\right]\|h\|_{L}^{\prime}
\end{aligned}
$$

Taking supremum to both hand sides we get

$$
\begin{aligned}
& \|B x(t)\| \leq \frac{1}{\Gamma(\alpha+1)}\|h\|_{L}^{1}+\left(\left|\frac{\delta_{1}}{1-\delta_{1}}\right|+\left|2\left(1-\delta_{1}\right)\right|+\left|2\left(1-\delta_{2}\right) \delta_{1}\right|\right)\left[\frac{1}{\Gamma \alpha+1}\|h\|_{L}^{1}\right] \\
& +\left(\left|\frac{\left(1-\delta_{1}\right)^{2} \delta_{2}^{2}}{\left(1-\delta_{1}\right)\left(1-\eta_{2} \delta_{2}\right)+\mid\left(1-\delta_{2}\right) \delta_{1} \eta_{1}}\right|\right. \\
& \left.+\left|\frac{\delta_{1} \eta_{1} \delta_{2}}{\left[\left(1-\delta_{1}\right)\left(1-\eta_{2} \delta_{2}\right)+\left(1-\delta_{2}\right) \delta_{1}\right]}\right|\right)\left[\frac{1}{\Gamma(p+1)}\|h\|_{L}^{1}\right] . \\
& =\frac{1}{\Gamma p+1}\|h\|_{L}^{1}\left(1+\left|\frac{\delta_{1}}{1-\delta_{1}}\right|+\left|2\left(1-\delta_{1}\right)\right|+\left|2\left(1-\delta_{2}\right) \delta_{1}\right|\right. \\
& \left.+\left|\frac{\left(1-\delta_{1}\right)^{2} \delta_{2}^{2}}{\left(1-\delta_{1}\right)\left(1-\eta_{2} \delta_{2}\right)+\mid\left(1-\delta_{2}\right) \delta_{1} \eta_{1}}\right|+\left|\frac{\delta_{1} \eta_{1} \delta_{2}}{\left[\left(1-\delta_{1}\right)\left(1-\eta_{2} \delta_{2}\right)+\left(1-\delta_{2}\right) \delta_{1}\right]}\right|\right) \\
& =\frac{l}{\Gamma(p+1)}\|h\|_{L}^{1} \\
& \|B x(t)\| \leq \frac{l}{\Gamma(p+1)}\|h\|_{L}^{1} .
\end{aligned}
$$

Where

$$
\begin{aligned}
& l=1+\left|\frac{\delta_{1}}{1-\delta_{1}}\right|+\left|2\left(1-\delta_{1}\right)\right|+\left|2\left(1-\delta_{2}\right) \delta_{1}\right| \\
& +\left|\frac{\left(1-\delta_{1}\right)^{2} \delta_{2}^{2}}{\left(1-\delta_{1}\right)\left(1-\eta_{2} \delta_{2}\right)+\mid\left(1-\delta_{2}\right) \delta_{1} \eta_{1}}\right| \\
& \left.+\left|\frac{\delta_{1} \eta_{1} \delta_{2}}{\left[\left(1-\delta_{1}\right)\left(1-\eta_{2} \delta_{2}\right)+\left(1-\delta_{2}\right) \delta_{1}\right]}\right|\right)
\end{aligned}
$$

for all $x \in S$, so $B$ is uniformly bounded on $S$. Now let $t_{1}, t_{2} \in J$, for any $x \in S$ one has

$$
\begin{aligned}
\mid B x\left(t_{1}\right)- & \left.B x\left(t_{2}\right)\right)|=| I^{\alpha} h\left(t_{1}\right)+\frac{\delta_{1}}{1-\delta_{1}}\left(I^{\alpha} h\left(\eta_{1}\right)\right) \\
& +\frac{\left(1-\delta_{1}\right) \delta_{2}}{\left(1-\delta_{1}\right)\left(1-\eta_{2} \delta_{2}\right)+\left(1-\delta_{2}\right) \delta_{1} \eta_{1}} \times \\
& {\left[\left(1-\delta_{1}\right) \delta_{2} I^{\alpha} h\left(\eta_{2}\right)-\left(1-\delta_{1}\right) I^{\alpha} h\left(\eta_{1}\right)-\left(1-\delta_{2}\right) \delta_{1} I^{\alpha} h\left(\eta_{1}\right)\right] } \\
& +\frac{\delta_{1} \eta_{1}}{\left(1-\delta_{1}\right)\left[\left(1-\delta_{1}\right)\left(1-\eta_{2} \delta_{2}\right)+\left(1-\delta_{2}\right) \delta_{1}\right]}\left[\left(1-\delta_{1}\right) \delta_{2} I^{\alpha} h\left(\eta_{2}\right)\right. \\
& \left.-\left(1-\delta_{1}\right) I^{\alpha} h\left(\eta_{1}\right)-\left(1-\delta_{2}\right) \delta_{1} I^{\alpha} h\left(\eta_{1}\right)\right] \\
& -I^{\alpha} h\left(t_{2}\right)-\frac{\delta_{1}}{1-\delta_{1}}\left(I^{\alpha} h\left(\eta_{1}\right)\right) \\
& -\frac{\left(1-\delta_{1}\right) \delta_{2}}{\left(1-\delta_{1}\right)\left(1-\eta_{2} \delta_{2}\right)+\left(1-\delta_{2}\right) \delta_{1} \eta_{1}}\left[\left(1-\delta_{1}\right) \delta_{2} I^{\alpha} h\left(\eta_{2}\right)\right. \\
& \left.-\left(1-\delta_{1}\right) I^{\alpha} h\left(\eta_{1}\right)-\left(1-\delta_{2}\right) \delta_{1} I^{\alpha} h\left(\eta_{1}\right)\right] \\
& -\frac{\delta_{1} \eta_{1} k}{\left(1-\delta_{1}\right)\left[\left(1-\delta_{1}\right)\left(1-\eta_{2} \delta_{2}\right)+\left(1-\delta_{2}\right) \delta_{1}\right]}\left[\left(1-\delta_{1}\right) \delta_{2} I^{\alpha} h\left(\eta_{2}\right)\right. \\
& \left.-\left(1-\delta_{1}\right) I^{\alpha} h\left(\eta_{1}\right)-\left(1-\delta_{2}\right) \delta_{1} I^{\alpha} h\left(\eta_{1}\right)\right] \mid, \\
& =\left|I^{\alpha} h\left(t_{1}\right)-I^{\alpha} h\left(t_{2}\right)\right| .
\end{aligned}
$$

As, we know that

$$
\begin{aligned}
I^{\alpha} h\left(t_{1}\right)-I^{\alpha} h\left(t_{2}\right) \mid & =\frac{1}{\Gamma(\alpha)} \mid \int_{0}^{t_{1}}\left(t_{1}-s\right)^{\alpha-1} g\left(s, x(s), I^{\alpha} x(s)\right) d s \\
& -\int_{0}^{t_{2}}\left(t_{2}-s\right)^{\alpha-1} g\left(s, x(s), I^{\alpha} x(s)\right) d s \mid \\
& \leq \frac{1}{\Gamma((\alpha))} \mid \int_{0}^{t_{1}}\left(t_{1}-s\right)^{(\alpha)-1} g\left(s, x(s), I^{\alpha} x(s)\right) d s \\
& -\int_{0}^{t_{1}}\left(t_{2}-s\right)^{(\alpha)-1} g\left(s, x(s), I^{\alpha} x(s)\right) d s \mid \\
& +\frac{1}{\Gamma((\alpha))} \mid \int_{0}^{t_{1}}\left(t_{1}-s\right)^{(\alpha)-1} g\left(s, x(s), I^{\alpha} x(s)\right) d s \\
& -\int_{0}^{t_{2}}\left(t_{2}-s\right)^{\alpha-1} g\left(s, x(s), I^{\alpha} x(s)\right) d s \mid \\
& \leq \frac{\|h\|_{L}^{1}}{\Gamma(\alpha)}\left(\left|\int_{0}^{t_{1}}\left[\left(t_{1}-s\right)^{\alpha-1}-\left(t_{2}-s\right)^{\alpha-1}\right] d s\right|\right. \\
& \left.+\mid \int_{t_{1}}^{t_{2}}\left(t_{2}-s\right)^{\alpha-1} d s\right) \\
& \leq \frac{\|h\|_{L}^{1}}{\Gamma(\alpha+1)}\left(\left|t_{1}^{\alpha}-t_{2}^{\alpha}\right|+\left|\left(t_{2}-t_{1}\right)^{p}\right|\right) .
\end{aligned}
$$

Since $t^{\alpha}$ is uniformly continuous on $J$, for $1<\alpha<2$, for any $\varepsilon>0$ there exist $\delta_{1}>0$ such that if $\left|\left(t_{1}-t_{2}\right)\right|<\delta_{1}$, we have

$$
\left|\left(t_{1}^{\alpha}-t_{2}^{\alpha}\right)\right|<\frac{\Gamma(\alpha+1)}{2\|h\|_{L}^{1}} \varepsilon
$$

Let $\delta=\min \left(\delta_{1},\left(\frac{\Gamma(\alpha+1)}{\left.2||\right|_{l} ^{\mid}} \varepsilon\right)^{\frac{1}{p}}\right)$, if $\left|t_{2}-t_{1}\right|<\delta$, we have 


$$
\left.\mid B x\left(t_{1}\right)-B x\left(t_{2}\right)\right) \mid<\frac{\|h\|_{L}^{1}}{\Gamma(\alpha+1)}\left(\frac{\Gamma(\alpha+1)}{2\|h\|_{L}^{1}} \varepsilon+\frac{\Gamma(\alpha+1)}{2\|h\|_{L}^{1}} \varepsilon\right)=\varepsilon
$$

This implies that $B(S)$ is equi-continuous. Thus, $B$ is completely continuous on $S$.

To prove hypothesis $\left(C_{3}\right)$ of Theorem 2.6, let $x \in X$ and $y \in S$ such that $x=A x+B y$, by assumptions $(H 1)$ and $(H 2)$, we have

$$
\begin{aligned}
& |x(t)| \leq|A x(t)|+|B y(t)| \\
& =\mid f(t, x(t))+\frac{\delta_{1}}{1-\delta_{1}}\left[f \left(\eta_{1}, x\left(\eta_{1}\right)+\frac{\eta_{1}}{\left(1-\delta_{1}\right)\left(1-\eta_{2} \delta_{2}\right)+\left(1-\delta_{2}\right) \delta_{1}}\left[( 1 - \delta _ { 1 } ) \delta _ { 2 } \left(f\left(\eta_{2}, x\left(\eta_{2}\right)\right)\right.\right.\right.\right. \\
& \left.\left.-\left(1-\delta_{1}\right) f(1, x(1))-\left(1-\delta_{2}\right) \delta_{1} f\left(\eta_{1}, x\left(\eta_{1}\right)\right)\right]\right)+\frac{\left(1-\delta_{1}\right) \delta_{2}}{\left(1-\delta_{1}\right)\left(1-\eta_{2} \delta_{2}\right)+\left(1-\delta_{2}\right) \delta_{1} \eta_{1}} \times \\
& {\left[\left(1-\delta_{1}\right) \delta_{2}\left(f\left(\eta_{2}, x\left(\eta_{2}\right)\right)-\left(1-\delta_{1}\right) f(1, x(1))-\left(1-\delta_{2}\right) \delta_{1} f\left(\eta_{1}, x\left(\eta_{1}\right)\right)\right] \mid\right.} \\
& +\mid I^{\alpha} h(t)+\frac{\delta_{1}}{1-\delta_{1}}\left(I^{\alpha} h\left(\eta_{1}\right)\right)+\frac{\left(1-\delta_{1}\right) \delta_{2}}{\left(1-\delta_{1}\right)\left(1-\eta_{2} \delta_{2}\right)+\left(1-\delta_{2}\right) \delta_{1} \eta_{1}} \times \\
& {\left[\left(1-\delta_{1}\right) \delta_{2} I^{a} h\left(\eta_{2}\right)-\left(1-\delta_{1}\right) I^{a} h\left(\eta_{1}\right)-\left(1-\delta_{2}\right) \delta_{1}{ }^{a} h\left(\eta_{1}\right)\right]+} \\
& \frac{\delta_{1} \eta_{1}}{\left(1-\delta_{1}\right)\left[\left(1-\delta_{1}\right)\left(1-\eta_{2} \delta_{2}\right)+\left(1-\delta_{2}\right) \delta_{1}\right]}\left[\left(1-\delta_{1}\right) \delta_{2} I^{\alpha} h\left(\eta_{2}\right)-\left(1-\delta_{1}\right) I^{\alpha} h\left(\eta_{1}\right)-\left(1-\delta_{2}\right) \delta_{1}{ }^{a} h\left(\eta_{1}\right)\right] \mid . \\
& \left.|A x(t)|=\mid f(t, x(t))+\frac{\delta_{1}}{1-\delta_{1}}-\frac{\delta_{1}}{1-\delta_{1}} \frac{\eta_{1}}{\left(1-\delta_{1}\right)\left(1-\eta_{2} \delta_{2}\right)+\left(1-\delta_{2}\right) \delta_{1}}\left(1-\delta_{2}\right) \delta_{1}\right) \\
& \left.-\frac{\left(1-\delta_{1}\right) \delta_{2}}{\left(1-\delta_{1}\right)\left(1-\eta_{2} \delta_{2}\right)+\left(1-\delta_{2}\right) \delta_{1} \eta_{1}}\left(1-\delta_{2}\right) \delta_{1}\right] f\left(\eta_{1}, x\left(\eta_{1}\right)\right) \\
& -\left[\frac{\left(1-\delta_{1}\right)^{2} \delta_{2}}{\left(1-\delta_{1}\right)\left(1-\eta_{2} \delta_{2}\right)+\left(1-\delta_{2}\right) \delta_{1} \eta_{1}}+\frac{\delta_{1} \eta_{1}}{\left(1-\delta_{1}\right)\left(1-\eta_{2} \delta_{2}\right)+\left(1-\delta_{2}\right) \delta_{1}}\right] f(1, x(1)) \\
& +\left[\frac{\delta_{1} \eta_{2} \delta_{2}}{\left(1-\delta_{1}\right)\left(1-\eta_{2} \delta_{2}\right)+\left(1-\delta_{2}\right) \delta_{1}}+\frac{\left(1-\delta_{1}^{2}\right)_{2}^{2}}{\left(1-\delta_{1}\right)\left(1-\eta_{2} \delta_{2}\right)+\left(1-\delta_{2}\right) \delta_{1}}\right] f\left(\eta_{2}, x\left(\eta_{2}\right)\right) \mid \\
& \leq|f(t, x(t))-f(t, 0)|+|f(t, 0)|+\left|\frac{\delta_{1}}{1-\delta_{1}}-\frac{\delta_{1}}{1-\delta_{1}} \frac{\eta_{1}}{\left(1-\delta_{1}\right)\left(1-\eta_{2} \delta_{2}\right)+\left(1-\delta_{2}\right) \delta_{1}}\left(1-\delta_{2}\right) \delta_{1}\right| \\
& \left.-\frac{\left(1-\delta_{1}\right) \delta_{2}}{\left(1-\delta_{1}\right)\left(1-\eta_{2} \delta_{2}\right)+\left(1-\delta_{2}\right) \delta_{1} \eta_{1}}\left(1-\delta_{2}\right) \delta_{1}\right]\left(f f\left(\eta_{1}, x\left(\eta_{1}\right)-f\left(\eta_{1}, 0\right)|+| f\left(\eta_{1}, 0\right) \mid\right)\right. \\
& +\frac{\left(1-\delta_{1}\right)^{2} \delta_{2}}{\left(1-\delta_{1}\right)\left(1-\eta_{2} \delta_{2}\right)+\left(1-\delta_{2}\right) \delta_{1} \eta_{1}}+\frac{\delta_{\gamma_{1}}}{\left(1-\eta_{1}\right)\left(1-\eta_{2} \delta_{2}\right)+\left(1-\delta_{2}\right) \delta_{1}}\{f(1, x(1)-f(1,0)|+| f(1,0) \mid) \\
& +\frac{\delta_{1} \eta_{2} \delta_{2}}{\left(1-\delta_{1}\right)\left(1-\eta_{2} \delta_{2}\right)+\left(1-\delta_{2}\right) \delta_{1} \eta_{1}}+\frac{\left(1-\delta_{1}\right)^{2} \delta_{2}^{2}}{\left(1-\delta_{1}\right)\left(1-\eta_{2} \delta_{2}\right)+\left(1-\delta_{2}\right) \delta_{1} \eta_{1}} \mid f\left(\eta_{2}, x\left(\eta_{2}\right)-f\left(\eta_{2}, 0\right)|+| f\left(\eta_{2}, 0\right) \mid\right) \\
& \leq\left|\frac{L|x(t)|)}{2(M+|x(t)|)}+F_{\mathrm{p}}\right|+\mid\left[\frac{\delta_{1}}{1-\delta_{1}}-\frac{\delta_{1}}{1-\delta_{1}} \frac{\eta_{1}}{\left(1-\delta_{1}\right)\left(1-\eta_{2} \delta_{2}\right)+\left(1-\delta_{2}\right) \delta_{1}}\left(1-\delta_{2}\right) \delta_{1}\right) \\
& \left.\left.-\frac{\left(1-\delta_{1}\right) \delta_{2}}{\left(1-\delta_{1}\right)\left(1-\eta_{2} \delta_{2}\right)+\left(1-\delta_{2}\right) \delta_{1} \eta_{1}}\left(\left(1-\delta_{2}\right) \delta_{1}\right)\right] \frac{L\left(x\left|x\left(\eta_{1}\right)\right|\right)}{2\left(M+\left|x\left(\eta_{1}\right)\right|\right)}+F_{0}\right) \mid \\
& +\left|\frac{\left(1-\delta_{1}\right)^{2} \delta_{2}}{\left(1-\delta_{1}\right)\left(1-\eta_{2} \delta_{2}\right)+\left(1-\delta_{2}\right) \delta_{1} \eta_{1}}+\frac{\delta_{1} \eta_{1}}{\left(1-\delta_{1}\right)\left(1-\eta_{2} \delta_{2}\right)+\left(1-\delta_{2}\right) \delta_{1}}\left(\frac{L|x(1)|)}{2(M+|x(1)|}+F_{0}\right)\right| \\
& +\left[\frac{\delta_{1} \eta_{1} \delta_{2}}{\left(1-\delta_{1}\right)\left(1-\eta_{2} \delta_{2}\right)+\left(1-\delta_{2}\right) \delta_{1}}+\frac{\left(1-\delta_{1}\right)^{2} \delta_{2}^{2}}{\left(1-\delta_{1}\right)\left(1-\eta_{2} \delta_{2}\right)+\left(1-\delta_{2}\right) \delta_{1}}\right]\left(\frac{\left.L\left|x\left(\eta_{1}\right)\right|\right)}{2\left(M+\left|x\left(\eta_{1}\right)\right|\right)}+F_{0}\right) \\
& \leq\left(L+F_{0}\right)+\mid\left[\frac{\delta_{1}}{1-\delta_{1}}-\frac{\delta_{1}}{1-\delta_{1}} \frac{\eta_{1}}{\left(1-\delta_{1}\right)\left(1-\eta_{2} \delta_{2}\right)+\left(1-\delta_{2}\right) \delta_{1}}\left(1-\delta_{2}\right) \delta_{1}\right) \\
& \left(1-\delta_{1}\right) \delta_{2} \\
& \left.-\frac{\left(1-\delta_{1}\right) \delta_{2}}{\left(1-\delta_{1}\right)\left(1-\eta_{2} \delta_{2}\right)+\left(1-\delta_{2}\right) \delta_{1} \eta_{1}}\left(1-\delta_{2}\right) \delta_{1}\right]\left(L+F_{0}\right) \\
& +\left|\left[\frac{\left(1-\delta_{1}^{2} \delta_{2}\right.}{\left(1-\delta_{1}\right)\left(1-\eta_{2} \delta_{2}\right)+\left(1-\delta_{2}\right) \delta_{1}}+\frac{\delta_{1} \eta_{1}}{\left(1-\delta_{1}\right)\left(1-\eta_{2} \delta_{2}\right)+\left(1-\delta_{2}\right) \delta_{1}}\right]\left(L+F_{0}\right)\right| \\
& +\left[\frac{\delta_{1} \gamma_{2} \delta_{2}}{\left(1-\delta_{1}\right)\left(1-\eta_{2} \delta_{2}\right)+\left(1-\delta_{2}\right) \delta_{1}}+\frac{\left(1-\delta_{1}\right)^{2} \delta_{2}^{2}}{\left(1-\delta_{1}\right)\left(1-\eta_{2} \delta_{2}\right)+\left(1-\delta_{2}\right) \delta_{1}}\right]\left(L+F_{0}\right) \\
& \leq\left(L+F_{0}\right)\left[1+\left[\frac{\delta_{1}}{1-\delta_{1}}-\frac{\delta_{1}}{1-\delta_{1}} \frac{\eta_{1}}{\left(1-\delta_{1}\right)\left(1-\eta_{2} \delta_{2}\right)+\left(1-\delta_{2}\right) \delta_{1}}\left(1-\delta_{2}\right) \delta_{1}\right)\right. \\
& \left.\frac{\left(1-\delta_{1}\right) \delta_{2}}{\left(1-\eta_{2} \delta_{2}\right)+\left(1-\delta_{2}\right) \delta_{1} \eta_{1}}\left(1-\delta_{2}\right) \delta_{1}\right] \\
& +\left[\frac{\left(1-\delta_{1}\right)^{2} \delta_{2}}{\left(1-\delta_{1}\right)\left(1-\eta_{2} \delta_{2}\right)+\left(1-\delta_{2}\right) \delta_{1}}+\frac{\delta_{1} \eta_{1}}{\delta \eta_{1} \delta}+\frac{\left(1-\delta_{1}\right)\left(1-\eta_{2} \delta_{2}\right)+\left(1-\delta_{2}\right) \delta_{1}}{\left(1-\delta_{1}\right)^{2} \delta_{2}^{2}}\right] \\
& \left.+\left[\frac{\delta_{\eta} \eta_{i} \delta_{2}}{\left(1-\delta_{1}\right)\left(1-\eta_{2}, \delta_{2}\right)+\left(1-\delta_{2}\right) \delta_{2}}+\frac{\left(1-\delta_{\delta^{2}}\right)^{2} \delta_{2}^{2}}{\left(1-\delta_{1}\right)\left(1-\eta_{2} \delta_{2}\right)+\left(1-\delta_{2}\right) \delta_{1}}\right]\right] \\
& =M_{1}\left(L+F_{0}\right)
\end{aligned}
$$

Also, we know

$$
\|B x(t)\| \leq \frac{l}{\Gamma(\alpha+1)}\|h\|_{L}^{1}
$$

Thus, we can write

$$
|x(t)| \leq M_{1}\left(L+F_{0}\right)+\frac{l}{\Gamma(\alpha+1)}\|h\|_{L}^{\mid} \leq N .
$$

\section{EXAMPLE}

Example 4.1 Consider the following coupled system of HFDEs

$$
\left\{\begin{array}{l}
D^{\ddagger}\left[x(t)-\left(\frac{t^{2}}{40}+\frac{e^{-1}|x(t)|}{10+|x(t)|}\right)\right]=\frac{t^{2}}{10}+y(t)+\cos \left|I^{\ddagger} y(t)\right|, \quad t \in[0,1], \quad \text { (4.1) } \\
D^{\ddagger}\left[y(t)-\left(\frac{t^{2}}{40}+\frac{e^{-t}|y(t)|}{10+|y(t)|}\right)\right]=\frac{t^{2}}{10}+x(t)+\cos \left|I^{\ddagger} x(t)\right|, \quad t \in[0,1], \\
x(0)=\frac{1}{2} x\left(\frac{1}{2}\right), x(1)=\frac{1}{3} x\left(\frac{1}{3}\right) \\
y(0)=\frac{1}{2} y\left(\frac{1}{2}\right), y(1)=\frac{1}{3} y\left(\frac{1}{3}\right) .
\end{array}\right.
$$

The solution of the BVP is given by

$$
L=1, M=10, \alpha=\frac{3}{2}, F_{0}=\sup |f(t, 0)|=\frac{1}{40},\|h\|=\frac{1}{30}, l=4.5 .
$$

Therefore $\left.M\left(L+F_{0}\right)+\frac{\| h_{L}}{\Gamma\left(\frac{3}{2}+1\right.}\right)<12$

So $N=12$. Hence, by Theorem 3.2, we conclude that the problem (4.1) has a solution in $\{(x, y):\|(x, y)\| \leq 12\}$.

\section{REFERENCES}

[1] Burton, T.A., 1998. A fixed-point theorem of Krasnoselskii. Applied Mathematics Letters, 11, 85-88.

[2] Khan, J.A., Zahoor, R.M.A., Qureshi, I.M. 2009.Swarm intelligence for the problem of non-linear ordinary differential equations and its application to well-known Wessinger's equation. European Journal of Scientific Research, 4, 514-525.

[3] Dhage, B.C. 2010. Quadratic perturbations of periodic boundary value problems of second order ordinary differential equations. Differential Equations and Applications, 2, 465-486.

[4] Dhage, B.C. 2009. Nonlinear quadratic first order functional integro-differential equations with periodic boundary conditions. Dynamic Systems and Applications, 18, 303-322.

[5] Dhage, B.C., Karande, B.D. 2005. First order integro-differential equations in Banach algebras involving Caratheodory and discontinuous nonlinearities. Electronic Journal of Qualitative Theory of Differential Equations, 21.

[6] Dhage, B.C., O'Regan, B.D. 2000. A fixed point theorem in Banach algebras with applications to functional integral equations. Functional Differential Equations, 7, 259-267.

[7] Dhage, B.C., Salunkhe, S.N., Agarwal, R.P., Zhang, W. 2005. A functional differential equation in Banach algebras. Mathematical Inequalities and Applications, 8, 89-99.

[8] Dhage, B.C. 1994. On á-condensing mappings in Banach algebras. Journal of Advanced Mathematical Studies, 63, 146-152.

[9] Dhage, B.C., Lakshmikantham, V. 2010. Basic results on hybrid differential equations. Nonlinear Analysis: Hybrid Systems, 4, 414-424.

[10] Dhage, B.C. 2004. A nonlinear alternative in Banach algebras with applications to functional differential equations. Nonlinear functional analysis and applications, 8, 563-575.

[11] Dhage, B.C. 1999. Fixed point theorems in ordered Banach algebras and applications. Pan-American Mathematical Journal, 9, 93102.

[12] Afshari, H., Kalantari, S., Karapinar, E. 2015. Solution of fractional differential equations via coupled fixed point. Electron. Journal of Differential Equations, 286.

[13] Dhage, B.C., Jadhav, N.S. 2013. Basic results in the theory of hybrid differential equations with linear perturbations of second type. Tamkang Journal of Mathematics, 44, 171-186.

[14] Caputo, M. 1967. Linear models of dissipation whose $Q$ is almost independent, II. Journals - Royal Astronomical Society, 13, 529-539.

[15] Diethelm, K, Ford, N.J. 2002. Analysis of fractional differential equations.Journal of Mathematical Analysis and Applications, 265, 229248.

[16] Diethelm, K, Ford, N.J. 2004. Multi-order fractional differential equations and their numerical solution. Applied Mathematics and Computation, 154, 621-640.

[17] Wang, G., Zhang, L., Song, G. 2012. Boundary value problem of a nonlinear Langevin equation with two different fractional orders and impulses. Fixed Point Theory and Applications, 200. 
[18] Jleli, M., Karapinar, E., Samet, B. 2014. Positive solutions for multipoint boundary value problems for singular fractional differential equations. Journal of Applied Mathematics, 2014, Article ID 596123.

[19] De la Sen, M, 2011. About robust stability of Caputo linear fractional dynamic systems with time delays through fixed point theory. Fixed Point Theory and Applications, Article ID 867932.

[20] De la Sen, M. 2011. Positivity and stability of the solutions of Caputo fractional linear time-invariant systems of any order with internal point delays. Abstract and Applied Analysis, Article ID 161246.

[21] Al-sawalha, M.M., Shoaib, M. 2016. Reduced-order synchronization of fractional order chaotic systems with fully unknown parameters using modified adaptive control. Journal of Nonlinear Sciences and Applications, 9, 1815-1825.

[22] Caraballo, T, Diop, M.A., Ndiaye, A.A. 2014. Asymptotic behavior of neutral stochastic partial functional integro- differential equations driven by a fractional Brownian motion. Journal of Nonlinear Sciences and Applications, 7, 407-421.

[23] Lu, H., Sun, S., Yang, D., Teng, H. 2013. Theory of fractional hybrid differential equations with linear perturbations of second type. Boundary Value Problems, 23.
[23] Lu, H., Sun, S., Yang, D., Teng, H. 2013. Theory of fractional hybrid differential equations with linear perturbations of second type. Boundary Value Problems, 23.

[24] Gafiychuk, V., Datsko, B., Meleshko, V. 2008. Mathematical modeling of time fractional reaction-diffusion systems. Journal of Computational and Applied Mathematics, 220, 215-225.

[25] Gejii, V.D. 2009. Positive solutions of a system of non-autonomous fractional differential equations. Journal of Mathematical Analysis and Applications, 302, 56-64, 22, 64-69.

[26] Kilbas, A.A., Srivastava, H.M., Trujillo, J.J. 2006. Theory and Applications of Fractional Differential Equations. Elsevier, Amsterdam.

[27] Chang, S.S., Cho, Y.J., Huang, N.J. 1996. Coupled fixed point theorems with applications. Journal of the Korean Mathematical Society, 33, 575585.

[28] Bashiri, T. Vaezpour, S. M., and Park, C. 2016. A coupled fixed point theorem and application to fractional hybrid differential problems. Fixed Point Theory and Applications, 23. 